\title{
REVIEW
}

\section{Viruses with deletions in antiapoptotic genes as potential oncolytic agents}

\author{
Ta-Chiang Liu ${ }^{*, 1}$ and David Kirn ${ }^{*, 2,3}$ \\ ${ }^{1}$ Molecular Neurosurgery Laboratory, Massachusetts General Hospital and Harvard Medical School, MA, USA; ${ }^{2}$ Department \\ of Clinical Pharmacology, University of Oxford, UK; ${ }^{3}$ Jennerex Biotherapeutics, Inc., Mountain View, CA, USA
}

\begin{abstract}
Replication-selective oncolytic viruses have emerged as a new treatment platform for cancers. However, selectivity and potency need to be improved before virotherapy can become a standard treatment modality. In addition, mechanisms that can be incorporated to enable targeting a broad range of cancer types are highly desirable. Cancer cells are well known to have multiple blocks in apoptosis pathways. On the other hand, viruses have evolved to express numerous antiapoptotic genes to antagonize apoptosis induced upon infection. Viruses with deletions in antiapoptotic genes can therefore be complemented by antiapoptotic genetic changes in cancer cells for efficient replication and oncolysis. In this review, we summarize the recent development of this concept, the potential obstacles, and future directions for optimization.
\end{abstract}

Oncogene (2005) 24, 6069-6079. doi:10.1038/sj.onc.1208734

Keywords: oncolytic; virus; cancer; apoptosis; virology; gene therapy

\section{Introduction}

Although the term 'oncolytic virus' was first described early in the 20th century, it was not until last decade that the genetic mechanisms to achieve/improve tumor selectivity were first described (Kirn et al., 2001). The first engineered replication-selective virus was published in 1991; Martuza et al. (1991) described a HSV mutant with deletion in the thymidine kinase $(t k)$ gene that replicates specifically in dividing tumor cells. The first replication-selective adenovirus $d l 1520$ (also known as Onyx-015) had a deletion in the E1B-55K gene and at least partially targeted the p53 pathway that is frequently abnormal in cancers (Barker and Berk, 1987; Bischoff et al., 1996). The development of dl1520 was based on our understanding of tumor biology and virology that the replication of DNA viruses and the carcinogenesis involve inactivating similar cellular defense mechanisms (Kirn, 2000). This led to the subsequent discovery of an adenoviral mutant with deletion in the E1A-CR2 region (dl922-947), which achieved tumor selectivity by targeting $\mathrm{Rb}$ pathway

*Correspondence: T-C Liu; E-mail: tachiangliu@yahoo.com or D Kirn; E-mail: dkirn@jennerex.com defects present in almost all cancer cells (Heise et al., 2000). However, clinical trials with $d l 1520$ to date show limited efficacy when used as a single agent despite selectivity (Kirn, 2001a).

Other approaches include the use of transcriptional control by placing essential immediate early viral genes under the control of tumor- and/or tissue-specific promoter/enhancer elements (Kirn et al., 2001), the use of inherently tumor-selective viruses (e.g. Reovirus, NDV, VSV, etc.), and engineering ligands for tumorselective receptors into the virus coat (Coffey et al., 1998; Lorence et al., 1988; Stojdl et al., 2000; Wickham, 2003). These are interesting approaches and are subject to extensive reviews elsewhere (Kirn, 2000; Wickham, 2003).

As mentioned above, the currently utilized gene deletions conferring selectivity also frequently result in significantly reduced potency of the virus in tumors due to the multifunctional nature of many viral proteins. The deletion mutant $d l 1520$ showed an overall response rate of $14 \%$ in patients with refractory locally recurrent head and neck cancers when given as a single agent by intratumoral injection. Similar results have been reported with gene-deleted herpes viruses (e.g. G207, 1716) in Phase I trials. Therefore, new approaches targeting ubiquitous tumor-specific pathways that can enhance the potency and efficacy while maintaining (or improving) the selectivity are eagerly awaited. This will presumably require identification of viral gene mutations resulting in selectivity but not attenuation in cancer cells.

\section{Apoptosis: ubiquitously inhibited in cancer cells}

Apoptosis, or 'programmed cell death', is a form of cellular suicide that is widely observed in nature. This process can be triggered by a variety of stimuli, including cytokines, hormones, viruses, mutagenic chemicals, or irradiation. Apoptosis can be induced by two different mechanisms, namely the mitochondrial (intrinsic) signaling pathway and the death-receptor (extrinsic) signaling pathway (Hengartner, 2000). The mitochondrial pathway, activated via a variety of intracellular stresses, including cytokine deprivation, serum starvation, $\mathrm{UV}$ and $\gamma$-irradiation, oncogenic stimuli such as c-MYC overexpression, is triggered by proapoptotic members of the Bcl-2 family, initially by a 
6070

subset of these called the 'BH3-only subfamily' proteins because they possess only one of the Bcl-2 homology (BH) domains. These include Bid, Bim, Harikari, Noxa, and a number of others. In response to environmental cues, these proteins engage another set of proapoptotic Bcl-2 members, the Bax subfamily (which includes Bax, Bak, and Bok), loosely residing on the mitochondrial outer membranes or in the cytosol. The interaction causes the latter to oligomerize and insert into the mitochondrial membrane. Here the complex acts to trigger the sudden and complete release of cytochrome $c$ and other proteins from all of the mitochondria in the cell (Green and Reed, 1998).

The released cytochrome $c$ binds to Apaf-1, a cytosolic protein (Li et al., 1997). This induces Apaf-1 oligomerization, and the Apaf-1 'apoptosome' recruits and activates procaspase-9. The active caspase- 9 now recruits and activates the effector procaspase-3. Bcl-2 and Bcl-xL (and probably other antiapoptotic members of this family) block cell death by preventing the mitochondrial release of the intermembrane proteins, including cytochrome $c$ (Yang et al., 1997). Once the process has achieved the mitochondrial step, these antiapoptotic proteins have no effect.

In mammals, Bcl-2 has at least 20 relatives, all of which share at least one conserved $\mathrm{BH}$ domain. The antiapoptotic branch includes four other proteins: Bcl$\mathrm{xL}, \mathrm{Bcl}-\mathrm{w}, \mathrm{Mcl}-1$, and A1. These proteins function by binding to Apaf- 1 and thereby preventing cytochrome $c$ release and procaspase- 9 activation. There are two groups of $\mathrm{Bcl}-2$ proteins that promote apoptosis: the Bax and the BH3-only families. Both types of proapoptotic protein are required to initiate apoptosis: the BH3only proteins act as damage sensors and direct antagonists of the prosurvival proteins, whereas the Bax-like proteins act further downstream in mitochondrial disruption (Huang and Strasser, 2000).

Apoptosis can also be triggered from outside of the cell, generally following cell-cell contact, by a family of transmembrane proteins - called death receptors which belong to the tumor necrosis factor (TNF) family of receptors. For Fas (CD95; Apo 1) and TNF signaling, the two most well-studied pathways, the engagement of cognate ligands of the TNF superfamily members (e.g. TNF- $\alpha$, FasL, TRAIL, etc.) induces oligomerization of their respective death receptors (TNFR, Fas, TRAIL-R, etc.). In the case of TNF signaling, the resulting aggregated TNFR1 intracellular domain is recognized by the adaptor protein TRADD (TNF receptor-associated death domain), which recruits additional adaptor proteins RIP (receptor-interacting protein), TRAF2 (TNF-R-associated factor 2), and FADD (Fas-associated death domain). FADD is composed of an amino terminal death effector domain (DED) and a carboxyl terminal death domain (DD). These adaptor proteins recruit key enzymes to TNF-R1 that are responsible for initiating signaling events: TRAF2 recruits cIAP-1 (cellular inhibitor of apoptosis protein-1) and cIAP-2, two antiapoptosis proteins that also have ubiquitin protein ligase activity. TRAF-2 also activates MAP kinase and leads to activation of c-Jun
(Liu et al., 1996). FADD recruits the upstream DEDcontaining procaspase-8 to the receptor via homophilic DED-DED interactions. DED is a specific example of a more global homophilic interaction domain termed CARD (caspase recruitment domain), which is found in several caspases with large prodomains, including caspases-2, -8, -9, and -10 (Hofmann et al., 1997). Procaspase- 8 within this newly formed death-inducing signalling complex (DISC; composed of death ligands, death receptors, FADD, TRADD, and Procaspase- 8 or -10) then proteolytically autocleaves itself to the active form and initiates apoptosis by subsequent cleavage of downstream effector caspases (caspase-3, -6, and -7) (Hirata et al., 1998).

However, the intrinsic and extrinsic apoptosis signaling pathways do not necessarily function individually and independently. Crosstalk between the two pathways is common and can ensure that the apoptosis process is carried out efficiently (Roth and Reed, 2002). For instance, the activation of caspase- 8 or -10 through the extrinsic pathway can cleave Bid (a proapoptotic member of the Bcl2 family). Truncated Bid (tBid) then translocates to mitochondria, where it induces conformational changes of Bax and Bak with subsequent oligomerization and formation of pores in the membrane, causing the release of cytochrome $c$ and Smac/ DIABLO, activating caspase-9 and -3 . This amplifies apoptosis induction through the extrinsic pathway. In contrast, DNA damage can induce transcriptional upregulation of some death receptors, such as Fas and death receptor 5 (DR5), through p53-dependent as well as p53-independent mechanisms (Ozoren and El-Deiry, 2003). This upregulation increases cellular sensitivity to death receptor ligands. Thus, crosstalk exists between the intrinsic and extrinsic apoptosis signaling pathways. A simplified summary of the intrinsic and extrinsic pathways of apoptosis is shown in Figure 1.

Cancer cells are well known to have dysregulated apoptosis pathways. Disruption of the intrinsic apoptosis pathway is extremely common in cancer cells. Indeed, the p53 tumor suppressor gene is the most frequent mutated gene in human tumors, and loss of p53 function can both disable apoptosis and accelerate tumor development in knockout mice (Vogelstein et al., 2000). Moreover, functional mutations or altered expression of $\mathrm{p} 53$ downstream effectors (PTEN, Bax, Bak, and Apaf-1), or upstream regulators (ATM, Chk2, $\mathrm{Mdm} 2$, and $\mathrm{p} 14^{\mathrm{ARF}}$ ), occur in human tumors (Rampino et al., 1997; Di Cristofano and Pandolfi, 2000; Johnstone et al., 2002).

Given the importance of Bcl-2 family members in regulating the intrinsic apoptosis pathway, it is not surprising that these genes are altered in tumor samples (Coultas and Strasser, 2003). Overexpression of Bcl-2, for example, occurs in low-grade follicular lymphomas due to the chromosome 14:18 translocation. Bcl-2 overexpression also occurs in the majority of nonsmall-cell lung carcinoma, colon and breast cancers. Conversely, proapoptotic Bcl-2 proteins are inactivated in certain cancers and disruption of these genes also promotes tumorigenesis in mice. In addition, other 


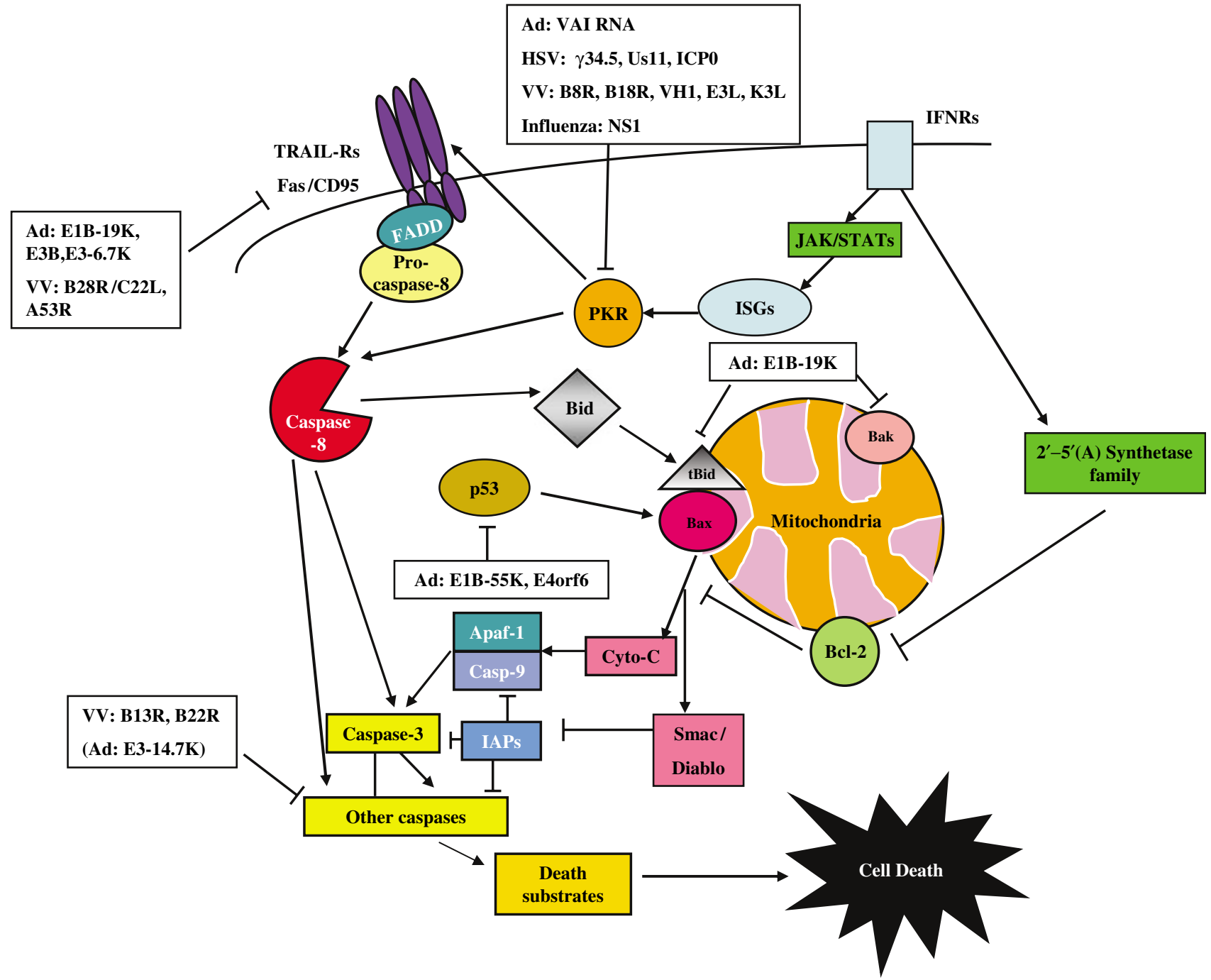

Figure 1 Apoptosis pathways. A simplified description on intrinsic, extrinsic apoptosis pathways as well as key players (e.g. interferon pathway and p53) and their interactions are included. Also included are the mechanisms that viruses employed to block apoptosis (modified from Roth W and Reed JC (2002) Nat. Med., 8, p. 217)

mechanisms are also involved in apoptosis pathway dysfunction in cancer cells. Amplified Akt and mutated PTEN have been found with high frequency in a variety of solid cancers, indicating the importance of this pathway in regulating tumorigenesis (Di Cristofano and Pandolfi, 2000).

Alterations that disrupt apoptosis downstream of the mitochondria have also been reported. For instance, silencing of Apaf-1 occurs in metastatic melanoma (Soengas et al., 2001), and overexpression of IAP family proteins, such as survivin or cIAP, is also common (Deveraux and Reed, 1999). Mutations or absence of nuclear translocation of certain caspases have also been demonstrated in several cancer cells. This implies that downstream defects in apoptosis contribute to oncogenesis.

Finally, defects in the death receptor apoptosis pathway also contribute to tumorigenesis. Tumor cells are often resistant to death-receptor-mediated apopto- sis, and mutations in Fas, TRAIL receptors, and downstream signaling pathways do occur in cancers (Ozoren and El-Deiry, 2003). Autoimmune lymphoproliferative syndrome (ALPS) is caused by germline mutations in CD95, resulting in inappropriate survival of activated T lymphocytes (Canale and Smith, 1967). ALPS patients have an increased incidence of lymphoma, possibly due to the expanded population of apoptosis-resistant $\mathrm{T}$ cells which can sustain further transforming mutations.

Escape from immune surveillance is important in solid tumors and non-T-cell leukemias. While cytotoxic lymphocytes predominantly kill tumor cells via the granule exocytosis pathway, FasL and TRAIL are also utilized. Thus, inactivation of the death receptor pathway could allow escape from immune surveillance and provide a survival advantage to developing tumor cells. In fact, loss of FasL or TRAIL function can promote tumor growth and metastasis (Hahne et al., 1996). 
Thus, apoptosis is regulated at many levels, including the initiation, transduction, amplification, and execution stages, and mutations that disrupt each of these stages have been detected in tumor cells. It is therefore believed that apoptosis pathways are dysregulated in virtually all human cancers. Table 1 lists examples of genetic blocks to cancer apoptosis pathways and the major associated cancer types.

\section{Virus infection and apoptosis}

In the course of a natural viral infection, viral particles or viral gene expression can also induce apoptosis, thereby limiting infection to the infected cells and preventing further viral replication and spread. However, viruses have evolved several strategies to block apoptosis of infected cells to promote viral replication (as described below). Since tumor cells have multiple blocks in the apoptosis pathways while those pathways in normal cells are intact, deleting viral antiapoptotic genes can, theoretically, render infected normal cells more susceptibility to apoptosis stimuli (thereby limiting toxicity), while the apoptosis induction in infected tumor cells will be minimally affected, thereby allowing the viral replication and oncolysis to proceed. Thus, viruses with deletions in antiapoptotic genes can be used as tumor-selective oncolytic agents. This is summarized in Figure 2. The antiapoptotic genes utilized by oncolytic viruses currently in development are summarized in Table 2 and Figure 1.

\section{Viral mutants that interfere with the TNF signaling pathway}

As described above, members of the TNF superfamily (CD95/FasL, TNF, TRAIL, etc.) play a major role in apoptosis induction in virus-infected cells. In the case of TNF- $\alpha$, it is secreted by macrophages and natural killer (NK) cells upon infection. It engages the TNF receptors (TNFRs) on cellular surface and ignites the apoptosis process. This limits the infection and is one of the major mechanisms the host immune system utilized for virus clearance. Adenovirus evolved several genes to antagonize this process (Burgert et al., 2002). Adenoviral E1B-19kD blocks TNF-mediated apoptosis mainly by binding to Bax and Bak, both of which belong to proapoptotic Bcl-2 family. The E1B-19kD (-) mutants first recognized by their ability to produce large plaques (lp phenotype), were cytocidal (cyt phenotype), and can induce host and viral DNA degradation (deg phenotype) (Chinnadurai et al., 1979). This efficient apoptosis induction was correlated with a reduced viral yield in certain cell lines (Chinnadurai et al., 1979; White et al., 1984). However, we and others have demonstrated that an adenoviral mutant with deletion in E1B-19kD gene has superior replication in a panel of cancer cells of different origin (Telling et al., 1994; Sauthoff et al., 2000; Kim et al., 2002; Liu et al., 2004). Although the exact mechanisms of the conflict is unclear, it is likely that a certain degree of cell-type specificity exists; in addition, it is possible that the cell lines used in previous works - most of them HPV-contaminants - might have been affected by the HPV sequence integrated. On the

Table 1 Major blocks in cancer apoptosis pathways

\begin{tabular}{|c|c|c|}
\hline $\begin{array}{l}\text { Targets in apoptosis } \\
\text { pathways }\end{array}$ & $\operatorname{Mechanism}(s)$ & Major tumor types \\
\hline $\mathrm{p} 53$ & $\begin{array}{l}\text { Missense mutations ( } 80-90 \%) \text {, deletions, insertions, } \\
\text { nonsense, etc. }\end{array}$ & $\begin{array}{l}\text { Majority of all human cancers; esp. brain, breast, } \\
\text { adrenocortical, sarcoma, etc. }\end{array}$ \\
\hline MDM2 & Amplification & Sarcomas \\
\hline Bcl-2 & Overexpression & $\begin{array}{l}\text { Majority of all cancers; esp. colorectal, B-cell } \\
\text { lymphomas, undifferentiated nasopharyngeal, stomach, } \\
\text { non-small-cell lung cancer }\end{array}$ \\
\hline Bax & Point mutation, deletion & Colon, stomach \\
\hline PTEN & Mutation, loss of heterozygosity, methylation & Glioblastoma, bladder, endometrial \\
\hline Apaf-1 & Mutation & Melanoma, leukemia \\
\hline $\mathrm{p} 14^{\mathrm{ARF}}$ & Mutation & Melanoma, lung, leukemia \\
\hline CD95/Fas & Mutation, downregulation & Lymphoma, melanoma, esophageal, lung, bladder \\
\hline TRAIL-R & Mutation & Breast, nasopharyngeal \\
\hline FLIP & Overexpression & Melanoma \\
\hline Caspase- 8 & Silenced & Neuroblastoma \\
\hline IAP & Overexpression & $\begin{array}{l}\text { Lung, colon, breast, pancreas, prostate, high-grade } \\
\text { lymphoma }\end{array}$ \\
\hline
\end{tabular}




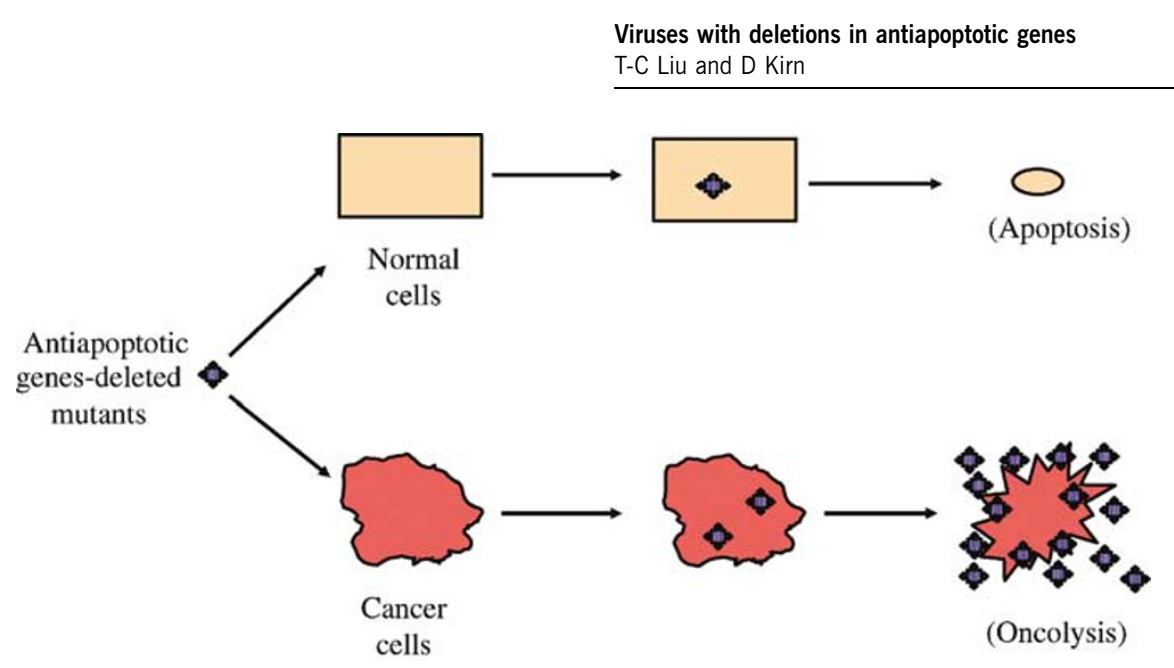

Figure 2 Viruses with deletions in antiapoptotic genes have enhanced selectivity. These viral mutants cannot replicate well in normal cells whose apoptosis pathways are intact; in contrast, viral replication and oncolysis can proceed in cancer cells which have multiple blocks in the apoptosis pathways

Table 2 Viral genes that antagonize apoptosis

\begin{tabular}{|c|c|c|}
\hline Approach & Example & Reference \\
\hline $\begin{array}{l}\text { Death signal ligand/re- } \\
\text { ceptor signaling }\end{array}$ & $\begin{array}{l}\text { Ad: E1B-19K, E3B, } \\
\text { E3-6.7K } \\
\text { VV: B28R/C22L, A53R } \\
\text { (soluble TNFRs) }\end{array}$ & $\begin{array}{l}\text { Jones and Shenk (1979); White et al. (1984); Moise et al. (2002) } \\
\text { Alcami et al. (1999) }\end{array}$ \\
\hline vBcl-2 & Ad: E1B-19K & Boyd et al., 1994 \\
\hline p53 inactivation & Ad: E1B-55K, E4 orf6 & Barker and Berk (1987); Dobner et al. (1996) \\
\hline IFN/PKR inactivation & $\begin{array}{l}\text { Ad: VAI RNA } \\
\text { HSV: } \gamma 34.5, \text { Us11, ICP0 } \\
\text { VV: B8R, B18R, VH1, } \\
\text { E3L, K3L } \\
\text { Influenza: NS1 }\end{array}$ & $\begin{array}{l}\text { Katze et al. (1987) } \\
\text { Leib et al. (1999); Peters et al. (2002); Hagglund and Roizman (2004) } \\
\text { Davies et al. (1993); Alcami and Smith (1995); Colamonici et al. (1995); } \\
\text { Najarro et al. (2001) } \\
\text { Zhirnov et al. (2002) }\end{array}$ \\
\hline Caspase inhibitors & $\begin{array}{l}\text { Ad: E3-14.7K } \\
\text { VV: B13R, B22R }\end{array}$ & $\begin{array}{l}\text { Chen et al. (1998) } \\
\text { Smith et al. (1989); Legrand et al. (2004) }\end{array}$ \\
\hline
\end{tabular}

other hand, normal primary cells infected with E1B$19 \mathrm{kD}$ mutant also showed an enhanced viral replication. However, when infected cells were treated with TNF- $\alpha$, no significant change of cytotoxicity or viral yield was observed in cancer cells. In contrast, the viral yield of E1B-19kD mutant was significantly reduced in normal primary cells after TNF treatment. This was correlated with a significant increase in apoptotic cell proportion (not observed in tumor cells even after TNF- $\alpha$ treatment). In vivo in immunocompetent tumor-bearing mice, systemic delivery of the mutant virus results in acute apoptosis induction in livers, which limits further viral replication and spread, while viral replication in tumors was not affected. Surprisingly, despite the acute apoptosis induction in livers, no animal death was found to be due to irreversible liver damage even at doses up to $10^{13}$ viral particles per injection (Liu et al., 2004). Of note, E1B-19kD also plays a role in limiting premature cell killing and viral spread (Chinnadurai, 1998). This independent property results in enhanced spread of the deletion mutant virus. TNF- $\alpha$ reduces viral spread in normal primary cells but not in tumor cells. Thus, adenoviral E1B-19kD mutants possess TNFmediated tumor selectivity.

Apart from E1B-19kD, several other adenoviral genes are capable of blocking TNF-induced cell death. Adenoviral E3B contains receptor internalization and degradation (RID) complex and E3-14.7, both of which have important functions in immunomodulation (Burgert et al., 2002). The RID complex, composed of RID $\alpha$ $(10.4 \mathrm{kDa})$ and $\operatorname{RID} \beta(14.5 \mathrm{kDa})$, localizes to the plasma membrane and intracellular vesicles. RID complex can internalize and degrade TNFR, RID as well as blocking TNF-mediated arachidonic acid release and cytosolic phospholipase A2 activation (Krajesi et al., 1996). RID has also been shown to antagonize apoptosis signaling from FasL and TRAIL as well as downregulating EGFR (Tollefson et al., 2001). E3-14.7kD binds to FIP (14.7 kDa interacting protein) family members to block TNF signaling, and it also inhibits arachidonic acid release.

As E3 genes have long been thought to be nonessential for viral replication, they are often deleted in 
both replication-deficient adenoviral vectors as well as replication-competent adenoviruses. Therefore, it is important to determine the impact of deleting these anitapoptotic genes in the context of gene therapy vector. We have tested one of these mutants with deletion in E3B (RID and E3-14.7kD). As predicted, the mutant showed TNF-mediated tumor selectivity in vitro, as seen with the E1B-19kD mutant. When administered intravenously to immunocompetent tumor-bearing mice, however, the persistence of the virus was decreased, both in tumor and normal tissues (Wang et al., 2003; Liu et al., 2004). Changing the administration route to intratumoral injection did not prolong the viral persistence in tumors. This was associated with significantly higher TNF- $\alpha$ expression. Therefore, E3B deletion enhances the selectivity, but at the same time decreases the persistence of the virus in vivo. It is unknown why E3B deletion and E1B-19kD deletion showed difference in persistence in vivo, but one possibility is that the RID complex and E3-14.7kD play a more important role than E1B-19kD in protecting infected tumor cells from TNF-mediated cell death/viral clearance in vivo. It is also possible that there are other unknown mechanisms of these genes. Of note, the fact that E3B-deleted virus was cleared more efficiently from the host may contribute to the low efficiency with the oncolytic adenoviruses in current clinical trials (Kirn, 2001a).

Deleting both E1B-19kD and E3B was relatively antagonistic. The double-deleted mutant showed reduced replication and cytopathic effects in normal cells in vitro and normal tissues in vivo; however, the replication/spread in tumors was intermediate between the two single deletion mutants. Among the three mutants, only the E1B-19kD mutant achieved a significant improvement in efficacy in animal model (Liu et al., in press).

Since tumor cells tend to have more blocks in intrinsic apoptosis pathway than in extrinsic pathway, deleting viral genes that target the intrinsic pathway (e.g. E1B19kD) might be optimal compared with those extrinsic pathway inhibitors (e.g. E3B). It will be interesting to see if this applies to other virus species as well.

Another E3 gene, E3-6.7kD, was recently shown to complex with RID to internalize TRAIL-R1, R2 and block TRAIL-induced apoptosis (Moise et al., 2002). Given the fact that TRAIL can selectively induces apoptosis in tumor cells, the above-mentioned adenoviral genes are excellent candidates for deletion to render more tumor-selective cell killing. However, the impact of deleting a single versus more than one of these genes needs to be studied to improve efficacy.

In terms of other currently available oncolytic virus species, vaccinia is another virus species that evolved anti-TNF mechanisms. Vaccinia virus encodes viral TNF receptors (vTNFRs) that resemble secreted versions of the extracellular domains of TNFRs and form functional oligomers that bind and sequester TNF (Alcami et al., 1999). Although the expression and activity of these vTNFRs varies greatly with different viral strains, at least three vaccinia virus strains have been shown to contain both soluble and cell-associated TNF-binding activities. It will therefore be interesting to test if vaccinia viruses with deletions in these genes can achieve TNF-mediated selectivity as seen with the adenoviral mutants described above.

\section{Viral mutants interfering with the intrinsic apoptosis pathway}

As mentioned earlier, adenovirus E1B-19kD is a functional homolog cellular Bcl-2 (Chiou et al., 1994). A region of limited sequence homology ( $\mathrm{BH}$ domain) has been identified between Bcl-2 and E1B-19kD. It has been shown that the capacity of adenovirus E1A to transform a cell relies on the expression of E1B-19kD (White and Cipriani, 1990). This is believed to be due to the apoptosis induction mediated by E1A, which requires a contemporary expression of $\mathrm{E} 1 \mathrm{~B}-19 \mathrm{kD}$ to antagonize the process. Some of the functions of E1B$19 \mathrm{kD}$ have been demonstrated to be complemented by cellular Bcl-2 overexpression, further supporting the notion that E1B-19kD functions as an antagonist in mitochondrial apoptosis pathway.

There are several levels where the interplay between the E1B-19kD protein and mitochondrial death signal propagation takes place. First, E1B-19kD can prevent Bak oligomerization and pore formation as well as Bakdependent Bax activation co-oligomerization and pore formation by direct binding to Bak. Second, by binding to Bax, E1B-19kD can prevent not only Bak-dependent Bax activation but also Bax activation through other mechanisms. Third, by binding to Bax, E1B-19kD may play a direct role in inhibiting Bax oligomerization. The phenotype of the E1B-19kD mutant adenovirus has been described above, although it remains interesting to explore the interaction of this mutant and cancer cells with defects in the mitochondrial pathway.

Virus encoded Bcl-2 homologs have also been reported for $\gamma-1$ herpes virus (EBV) and $\gamma$-2 herpes virus (HHV-8 and herpesvirus saimiri) (Tarodi et al., 1994; Cheng et al., 1997; Nava et al., 1997). However, there is no report on $\mathrm{v}-\mathrm{Bcl}-2$ in $\alpha$ herpes virus (HSV-1) or other viral species being used for oncolytic purposes.

\section{Viral mutants that interfere with the p53 pathway}

Adenoviral E1B-55kD has been shown to block p53induced apoptosis by direct binding to p53, thereby blocking its transcriptional activation. Since p53 is mutated in the majority of the human cancers, the mutant virus $d l 1520$ was thought to be selective for cancer treatment. Indeed, in the original report testing $d l 1520$ for oncolytic purposes, the status of cellular p53 was well documented; cancer cells with mutant p53 showed higher susceptibility and cytotoxicity to $d l 1520$ than their p53 normal counterpart (Bischoff et al., 1996). When tested in patients in clinical trials there was also a good correlation between response rate and 
mutant p53 gene sequences within head and neck cancers. Patients with mutant p53 within their tumor sample were more likely to respond to $d l 1520$ (Kirn, 2001b). Thus, $d l 1520$ was the first biologic agent described to preferentially target cancers with p53 deficiency. However, two problems arose: first of all, as E1B-55kD protein not only binds to p53 but also possesses other functions such as mediating viral transport and host protein synthesis shut-off, the deletion of this gene attenuated the virus. Viral yield was significantly reduced compared to control virus dl309, even in many p53-mutated cell lines. In addition, subsequent research also pointed out that the status of p53 was not the sole determinant of selectivity. Cell cycle status, as well as CAR and other cellular factors, play significant roles in determining the selectivity of this virus. As expected, the status of one of the proteins in the p53 induction pathway, p14 ${ }^{\mathrm{ARF}}$, was shown to be critical as well (Ries et al., 2000); deletion led to p53 deficiency and a minor increase in virus replication. Therefore, one argues that the function of the whole p53 pathway, rather than specific mutations of one or two key components, might be one of the determining factors for tumor selectivity. Of note, efforts have been made to improve the potency of $d l 1520$ while preserving its selectivity. Shen et al. (2001) described the use of a single amino-acid mutation in isolating an E1B-55kD mutant that did not bind to p53, but partially retained the late function of E1B-55kD. Such a mutant virus should theoretically have enhanced potency and efficacy over the original $d l 1520$.

Apart from E1B-55kD, adenoviral E4orf6 has been shown to play a role in downregulating p53. The E4orf6 protein binds to and form complex with both E1B-55kD and p53, and the complex formation facilitates p53 destabilization (Leppard and Everett, 1999). E4orf6 also interacts with several other viral proteins, some of which identified. However, to date, there is no report on adenoviral mutants with E4orf6 deletion targeting cancers with dysfunctioned p53 pathway.

\section{Viral mutants interfering with the IFN/PKR pathway}

Antiviral cytokine interferon (IFN) has been a frequent target for a panel of viral genes to evade immune surveillance. There are two types of interferons: type I IFNs include IFN- $\alpha$, IFN- $\beta$, IFN- $\omega$, and IFN- $\tau$, while type II IFN is IFN- $\gamma$. Type I IFNs are the major antiviral IFNs, and can be produced by most cell types, whereas IFN- $\gamma$ is only produced by certain cells of immune system (NK cells, CD4 $+\mathrm{T}$ helper cells, and CD8 + cytotoxic T cells). Type I IFNs are induced during viral infection by viral-replication products, such as double-stranded (ds)RNA. The secreted IFNs in turn bind to their specific cellular receptors and trigger the intracellular downstream pathway. The main pathway involved is the JAK-STAT pathway which upon activation induces the expression of several IFNstimulated genes. Among them, the most widely studied is the dsRNA-activated serine/threonine protein kinase, known as protein kinase R (PKR) (Katze et al., 2002). These genes then in turn regulate the antiviral, antiproliferative, and immunoregulatory responses. In addition, IFN also activates the $2^{\prime}, 5^{\prime}$-oligoadenylate synthetase - RNaseL pathway to induce apoptosis; this is separated from the PKR pathway.

Given the importance of IFNs in limiting viral infection in vivo, it is not surprising to find that many virus species encode genes to target the IFN/PKR pathway. Adenovirus encodes viral-associated type I RNA (VAI RNA) that blocks PKR signaling (Katze et al., 1987). Adenoviral mutant with deletion in VAI RNA takes advantage of the defective PKR pathway in Ras-transformed cancer cells and are selective for cancers with activated Ras (Cascallo et al., 2003). Since oncogenic Ras can induce endogenous PKR inhibitor, the PKR pathway in Ras-transformed cells is genetically blocked, thereby allowing the viral replication of the VAI mutant to proceed, while the lack of VAI RNA makes infected normal cells (in which the PKR pathway is intact) sensitive to IFN-mediated killing.

Herpes viruses utilize several strategies to block the IFN pathway. HSV ICP 0 blocks the JAK-STAT pathway and directly downregulates the level of expression of the IFN-stimulated genes (Mossman and Smiley, 2002). HSV Us11 is an inhibitor of PKR (Cassady and Gross, 2002). On the other hand, HSV $\gamma 34.5$ recruits cellular protein phosphatase $1 \alpha(\mathrm{PP} 1 \alpha)$ to dephosphorylate eukaryotic initiation factor $2, \alpha$-subunit (eIF-2 $\alpha$ ), which is downstream of PKR (Katze et al., 2002). Of note, recent studies have shown that the modulation of the IFN system by HSV, especially PKR, is the main mechanism for neurovirulence and pathogenesis associated with HSV infection. In addition, HSV also encodes $2^{\prime}, 5^{\prime}$-adenosine (A) derivatives to block the $2^{\prime}, 5^{\prime}$-oligoadenylate synthetase - RNaseL pathway (Moss et al., 1977).

Among the genes mentioned above, only $\gamma 34.5$ mutants have been reported for oncolytic purpose. HSV mutants with deletions in one or both copies of $\gamma$ 34.5 show no sign of neurotoxicity even when given intracranially (Todo et al., 2000; Bennett et al., 2002). $\gamma 34.5$ mutants efficiently eradicate tumors in various human tumor-nude mice xenograft systems, and evidence of viral persistence in brain tumors was documented in patients. However, $\gamma 34.5$ gene deletion also attenuates the virus. The viral yield of $\gamma 34.5$ mutants (e.g. G207, NV1020, etc.) was at least 10-fold lower than wild-type HSV (Mineta et al., 1995). This may be attributed to the premature protein synthesis shutoff by activated PKR.

Although no Us11 mutant was reported on oncolytic HSV construct, several publications indicated that the expression of Us11 before the activation of PKR can compensate for the $\gamma 34.5$ in preventing protein synthesis shut-off (Poppers et al., 2000). Since the Us11 gene is nonessential for HSV replication, it will be interesting to see if the deletion of both Us11 and $\gamma 34.5$ has any additional benefit. The other protein, ICP 0 , is multifunctional and plays a role in transactivating other HSV 
genes. ICP 0 deletion mutant showed defective replication in vitro at low multiplicity of infection (MOI), probably due to its multifunctional character (Hagglund and Roizman, 2004). Therefore, deleting ICP 0 does not seem to be feasible for oncolytic HSV.

As seen with the case of TNF- $\alpha$, vaccinia virus (and other members of poxvirus) encodes soluble IFN receptors (B8R and $\mathrm{B} 18 \mathrm{R}$ ) that block the binding of IFNs to their cellular receptors (Alcami and Smith, 1995). In addition, the vaccinia virus E3L protein can bind to dsRNA (which activates IFN secretion), sequester dsRNA molecules, and also directly inhibit the activation of PKR. The $\mathrm{K} 3 \mathrm{~L}$ protein encodes a eukaryotic initiation factor $2, \alpha$-subunit (eIF-2 $\alpha$ ) homolog that acts as a psudosubstrate for PKR, thereby blocking the PKR signaling (Carroll et al., 1993). Other mechanisms are also proposed for the E3L and $\mathrm{K} 3 \mathrm{~L}$ gene product to antagonize the IFN pathway. Vaccinia vector lacking $\mathrm{B} 8 \mathrm{R}$ was shown to be less toxic to immunocompetent and athymic mice (Verardi et al., 2001). In addition, no concomitant reduction in immunogenicity was observed. On the other hand, the viral yield of B18R mutant in cells pretreated with IFN was significantly reduced (Alcami et al., 2000). Vaccinia E3L protein, although nonessential for replication in chicken embryo fibroblasts, was found to have a severe impact in viral yield in several other cell lines (Beattie et al., 1996). Similarly, K3L mutant also showed hostrange restriction (Langland and Jacobs, 2002), although different from that of the E3L mutant. Interestingly, it has been proposed that both E3L and $\mathrm{K} 3 \mathrm{~L}$ were adopted by vaccinia to accommodate a broad host range. Therefore, more studies need to be done before incorporating $\mathrm{E} 3 \mathrm{~L}$ and/or $\mathrm{K} 3 \mathrm{~L}$ deletion for oncolytic vaccinia design.

Influenza virus utilizes two main mechanisms to block the IFN pathway. It activates the $\mathrm{p} 58^{\mathrm{IPK}}$-mediated hostcell stress-response pathway, and uses it against the host cell by directing $\mathrm{p} 58^{\mathrm{IPK}}$ to block the activation of PKR. In addition, the influenza virus nonstructural protein NS1 is a dsRNA-binding protein that can block the dsRNA-activated IFN expression. NS1 can also interact with PKR and inhibit its kinase function. There are also reports that NS1 can interfere with the IFN-regulatory factors (Zhirnov et al., 2002). Mutant influenza virus with deletion in NS1 has been shown to replicate in and kill Ras-transformed tumor cells which have deficient PKR pathways (Bergmann et al., 2001; Muster et al., 2004)

It is worth mentioning that some other virus species actually take advantage of the defective PKR pathway (through Ras transformation) and have been tested for oncolysis. These viruses are all RNA viruses, namely measles, mumps, reovirus, NDV, VSV, poliovirus, among others (Kirn et al., 2001). At the course of replication, these viruses produce dsRNA that inititates the IFN signaling pathway. Therefore in cancers where the PKR is blocked genetically, the replication of these viruses can proceed while that in normal cells is diminished due to intact PKR. Readers can refer to a recent article for more thorough review (Russell, 2002).

\section{Viral genes as caspase inhibitors}

Poxviruses have evolved several genes that inhibit caspases. Vaccinia virus B13R and B22R gene products show amino acid similarity to serine protease inhibitors (Smith et al., 1989). B13R can block apoptosis induced by Fas and TNF- $\alpha$ (Kettle et al., 1997). Caspase-1 (interleukin-1 $\beta$-converting enzyme), and presumably other caspases, has been shown to be inhibited by B13R protein. B22R presumably has a similar effect. Mutants with deletions in these genes show no difference in viral replication in tissue culture (Kettle et al., 1995). Although no significant difference in virulence was seen compared to wild-type virus in murine intranasal model, subsequent study showed that virulence was reduced in both athymic and immunocompetent mice (Legrand et al., 2004). Furthermore, immunogenicity was not reduced by evidence of humoral, $\mathrm{T}$-helper, and cytotoxic T-cell immune responses. However, B13R has a limited distribution among vaccinia strains; it is not detected in Copenhagen, Tian Tan, Lister, and Tashkent strains. In contrast, B22R is expressed in all vaccinia strains tested (Kettle et al., 1995). Nevertheless, it will be interesting to explore the effect of B13R and B22R in the context of oncolytic virus.

Although it has been proposed that adenovirus E3$14.7 \mathrm{kD}$ gene product might play a role in blocking the activity of caspases, only one report showed that the $14.7 \mathrm{kD}$ protein interacts with caspase- 8 , and blocks apoptosis mediated by Fas, FADD, or caspase-8 (Chen et al., 1998), while other researchers were not able to confirm the finding (McNees and Gooding, 2002). Therefore, it is still unclear whether E3-14.7kD acts as a direct caspase inhibitor.

\section{Problems and future directions}

\section{Exploring new viral gene combinations for deletion}

Viral antiapoptotic genes are potential candidates for improving the selectivity and potency of oncolytic viruses. Likewise, for viruses containing multiple antiapoptotic genes, deleting more than one of those genes might further improve the selectivity and potency. However, as these genes are often multifunctional, one must weigh between improving selectivity and losing some critical functions. For example, deleting both E1B$55 \mathrm{kD}$ and $\mathrm{E} 1 \mathrm{~B}-19 \mathrm{kD}$ genes did not improve the potency over E1B-19kD gene deletion in isolation (Kim et al., 2002). Similarly, an adenoviral mutant with deletions in both E1A-CR2 and E1B-55kD has been reported (Gomez-Manzano et al., 2004). Although selectivity was moderately improved, no significant improvement in potency and efficacy was noted. It is therefore important to determine if the viral genes of interest bear any other critical function in the course of viral infection. Of note, targeted deletions to knock out the antiapoptotic function, but not desired functions, should be explored. In addition, deletion of antiapoptotic gene(s) with other viral genes that do not attenuate 


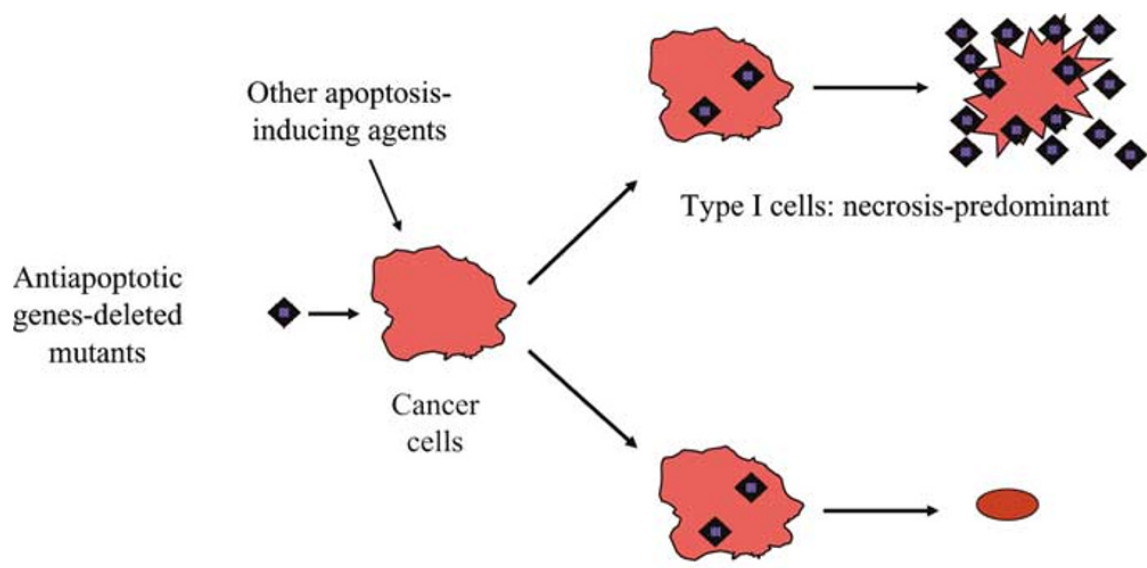

Type II cells: apoptosis-predominant

Figure 3 The fate of tumor cells infected with antiapoptotic genes-deleted oncolytic viruses. In type I cells, no defect in apoptosis pathway is complemented/restored, and viral replication proceeds. In type II cells, the apoptosis pathway is complemented by sensitization (due to virus infection and/or administration of apoptosis-inducing agents) and a simultaneous lack of antiapoptotic genes

the virus might greatly improve the selectivity as well as potency. For instance, deleting E1B-19kD and E1ACR2 might show superior effect compared with the single mutants.

Caution must be taken when designing new virus constructs. While deleting more than one antiapoptotic genes might increase the selectivity, a potential disadvantage is the attenuation in potency, even when the deleted viral genes do not possess other major functions. We have shown that adenoviral E1B-19kD(-)/E3B $(-)$ mutant was relatively more selective than E1B$19 \mathrm{kD}(-) / \mathrm{E} 3 \mathrm{~B}(+)$ and $\mathrm{E} 1 \mathrm{~B}-19 \mathrm{kD}(+) / \mathrm{E} 3 \mathrm{~B}(-)$ mutants. However, the antitumoral potency was largely reduced compared to the E1B-19kD(-)/E3B(+) mutant. Similarly, $\mathrm{E} 3 \mathrm{~B}(-)$ mutant showed reduced persistence in vivo believed to be due to reduced TNF- $\alpha$ antagonism which led to enhanced viral clearance (Liu et al., in press). On the other hand, in a mouse pneumonia model, E3B(-) mutant was shown to induce more significant inflammatory response than single E3B component (RID, E3$14.7 \mathrm{kD}$ ) mutants. Similarly, deleting genes that interfere with both TNF- and IFN-mediated apoptosis pathways might increase the selectivity, but it is also likely that the virus will be cleared more rapidly. Thus, more preclinical studies should be done to maximize the potency and selectivity. Of note, the use of immunocompetent tumor models is critical in examining the effect of these mutants as these genes often play a role in immunomodulation (Hallden et al., 2003).

\section{Cell type specificity?}

This also raises another important question: are tumor cells infected with multimutated viruses (or indeed, any of those single antiapoptotic gene-deleted mutants) more sensitive to apoptosis? While targeting only one player in a particular apoptosis pathway is probably not efficient enough to drive the infected cells to apoptosis, there are possibilities that some of the cell types might become more sensitive. Since tumors have various mechanisms blocking the apoptosis pathways, it is likely that defect in apoptosis pathways in some tumor types will complement replication of these mutant viruses, thereby supporting viral replication and spread, whereas other cell types might not be complementing. In fact, although most tumor cells infected with these mutants did not become more apoptosis-prone, some publications have identified a number of cells that do (White et al., 1992; Sauthoff et al., 2000). It seems likely that there are two main types of cells that respond differently to these mutants (Figure 3): the first type of cells (type I cells) are not affected in the apoptosis process. Therefore, replication-induced viral oncolysis is the major mechanism of cell death (necrosis-predominant). In contrast, in the second type of cells (type II cells) that become more apoptosis-prone upon infection, the mechanism antagonizing the apoptosis process is largely attenuated, and therefore more infected cells are killed due to infection-related apoptosis while less are killed through replication-mediated oncolysis (apoptosis-predominant). This will presumably be more evident when another apoptosis-inducing mechanism is induced (e.g. chemotherapy or apoptosis-inducing transgenes expression). Although type I cells are the ones that will support the replication of these oncolytic viruses better, type II cells can still be killed by these viruses, although through a different mechanism. Detailed molecular analysis on the defect of apoptosis pathways in each different tumor will help to predict the fate of the tumor receiving this type of treatment. Nevertheless, despite the low degree of viral replication and spread in type II cells, it is expected that patients with type II cancers can still benefit from these viruses.

\section{Combination with standard and alternative therapeutics}

Standard cancer gene therapy development strategy includes the combination of gene therapy with chemo-/ 
radio-therapeutic agents. Most of these agents, however, induce apoptosis. Since chemotherapeutic agents act on a wide variety of targets in apoptosis pathway, combining antiapoptotic genes-deleted oncolytic viruses with these agents will theoretically enhance the antitumoral efficacy. However, to maximize viral oncolysis and tumor apoptosis, the administration dosage and route need to be optimized for each agent, since rapid apoptosis induction might limit viral replication and spread. Other therapeutics such as antiangiogenesis or small molecules that target specific oncogenic pathways (e.g. Ras inhibitors, EGFR antagonists) will also be

\section{References}

Alcami A, Khanna A, Paul NL and Smith GL. (1999). J. Gen. Virol., 80, 949-959.

Alcami A and Smith GL. (1995). J. Virol., 69, 4633-4639.

Alcami A, Symons JA and Smith GL. (2000). J. Virol., 74, 11230-11239.

Barker DD and Berk AJ. (1987). Virology, 156, 107-121.

Beattie E, Kauffman EB, Martinez H, Perkus ME, Jacobs BL, Paoletti E and Tartaglia J. (1996). Virus Genes, 12, 89-94.

Bennett JJ, Delman KA, Burt BM, Mariotti A, Malhotra S, Zager J, Petrowsky H, Mastorides S, Federoff H and Fong Y. (2002). Cancer Gene Ther., 9, 935-945.

Bergmann M, Romirer I, Sachet M, Fleischhacker R, GarciaSastre A, Palese P, Wolff K, Pehamberger H, Jakesz R and Muster T. (2001). Cancer Res., 61, 8188-8193.

Bischoff JR, Kirn DH, Williams A, Heise C, Horn S, Muna M, Ng L, Nye JA, Sampson-Johannes A, Fattaey A and McCormick F. (1996). Science, 274, 373-376.

Boyd JM, Malstrom S, Subramanian T, Venkatesh LK, Schaeper U, Elangovan B, D'Sa-Eipper C and Chinnadurai G. (1994). Cell, 79, 341-351.

Burgert HG, Ruzsics Z, Obermeier S, Hilgendorf A, Windheim $\mathrm{M}$ and Elsing A. (2002). Curr. Top. Microbiol. Immunol., 269, 273-318.

Canale VC and Smith CH. (1967). J. Pediatr., 70, 891-899.

Carroll K, Elroy-Stein O, Moss B and Jagus R. (1993). J. Biol. Chem., 268, 12837-12842.

Cascallo M, Capella G, Mazo A and Alemany R. (2003). Cancer Res., 63, 5544-5550.

Cassady KA and Gross M. (2002). J. Virol., 76, 2029-2035.

Chen P, Tian J, Kovesdi I and Bruder JT. (1998). J. Biol. Chem., 273, 5815-5820.

Cheng EH, Nicholas J, Bellows DS, Hayward GS, Guo HG, Reitz MS and Hardwick JM. (1997). Proc. Natl. Acad. Sci. USA, 94, 690-694.

Chinnadurai G. (1998). Semin. Virol., 8, 399-408.

Chinnadurai G, Chinnadurai S and Brusca J. (1979). J. Virol., 32, 623-628.

Chiou SK, Tseng CC, Rao L and White E. (1994). J. Virol., 68, 6553-6566.

Coffey MC, Strong JE, Forsyth PA and Lee PW. (1998). Science, 282, 1332-1334.

Colamonici OR, Domanski P, Sweitzer SM, Larner A and Buller RM. (1995). J. Biol. Chem., 270, 15974-15978.

Coultas L and Strasser A. (2003). Semin. Cancer Biol., 13, $115-123$.

Davies MV, Chang HW, Jacobs BL and Kaufman RJ. (1993). J. Virol., 67, 1688-1692.

Deveraux QL and Reed JC. (1999). Genes Dev., 13, 239-252.

Di Cristofano A and Pandolfi PP. (2000). Cell, 100,387-390. good candidates for combination treatment since they have distinct mechanisms of action without crossresistance. In addition, these viruses can also be armed with therapeutic transgenes and further enhance the selectivity and potency.

In summary, deleting antiapoptotic genes in oncolytic viruses is an appealing approach which can lead to enhanced selectivity and/or potency. Future work exploring the genetic determinants for susceptibility to these mutants and incorporation of these mutations into multimutated viral mutants will help to improve the design of oncolytic viruses.

Dobner T, Horikoshi N, Rubenwolf S and Shenk T. (1996) Science, 272, 1470-1473.

Gomez-Manzano C, Balague C, Alemany R, Lemoine MG, Mitlianga P, Jiang H, Khan A, Alonso M, Lang FF, Conrad CA, Liu TJ, Bekele BN, Yung WK and Fueyo J. (2004). Oncogene, 23, 1821-1828.

Green DR and Reed JC. (1998). Science, 281, 1309-1312.

Hagglund R and Roizman B. (2004). J. Virol., 78, 2169-2178.

Hahne M, Rimoldi D, Schroter M, Romero P, Schreier M, French LE, Schneider P, Bornand T, Fontana A, Lienard D, Cerottini J and Tschopp J. (1996). Science, 274, 1363-1366.

Hallden G, Hill R, Wang Y, Anand A, Liu T, Lemoine NR, Francis J, Hawkins L and Kirn D. (2003). Mol. Ther., 8, 412-424.

Heise C, Hermiston T, Johnson L, Brooks G, SampsonJohannes A, Williams A, Hawkins L and Kirn D. (2000). Nat. Med., 6, 1134-1139.

Hengartner MO. (2000). Nature, 407, 770-776.

Hirata H, Takahashi A, Kobayashi S, Yonehara S, Sawai H, Okazaki T, Yamamoto K and Sasada M. (1998). J. Exp. Med., 187, 587-600.

Hofmann K, Bucher P and Tschopp J. (1997). Trends. Biochem. Sci., 22, 155-156.

Huang DC and Strasser A. (2000). Cell, 103, 839-842.

Johnstone RW, Ruefli AA and Lowe SW. (2002). Cell, 108, 153-164.

Jones N and Shenk T. (1979). Cell, 17, 683-689.

Katze MG, DeCorato D and Safer B. (1987). EMBO J., 6, 689-697.

Katze MG, He Y and Gale Jr M. (2002). Nat. Rev. Immunol., 2, 675-687.

Kettle S, Alcami A, Khanna A, Ehret R, Jassoy C and Smith GL. (1997). J. Gen. Virol., 78, 677-685.

Kettle S, Blake NW, Law KM and Smith GL. (1995). Virology, 206, 136-147.

Kim J, Cho JY, Kim JH, Jung KC and Yun CO. (2002). Cancer Gene Ther., 9, 725-736.

Kirn D. (2000). Oncogene, 6660-6668.

Kirn D. (2001a). Gene Therapy, 8, 89-98.

Kirn D. (2001b). Expert Opin. Biol. Ther., 1, 525-538.

Kirn D, Martuza RL and Zwiebel J. (2001). Nat. Med., 7, 781-787.

Krajcsi P, Dimitrov T, Hermiston TW, Tollefson AE, Ranheim TS, Vande Pol SB, Stephenson AH and Wold WS. (1996). J. Virol., 70, 4904-4913.

Langland JO and Jacobs BL. (2002). Virology, 299, 133-141.

Legrand FA, Verardi PH, Jones LA, Chan KS, Peng Y and Yilma TD. (2004). J. Virol., 78, 2770-2779.

Leib DA, Harrison TE, Laslo KM, Machalek MA, Moorman NJ and Virgin HW. (1999). J. Exp. Med., 189, 663-672. 
Leppard KN and Everett RD. (1999). J. Gen. Virol., 80, 997-1008.

Li P, Nijhawan D, Budihardjo I, Srinivasula SM, Ahmad M, Alnemri ES and Wang X. (1997). Cell, 91, 479-489.

Liu TC, Hallden G, Wang Y, Brooks G, Francis J, Lemoine N and Kirn D. (2004). Mol. Ther., 9, 786-803.

Liu ZG, Hsu H, Goeddel DV and Karin M. (1996). Cell, 87, 565-576.

Lorence RM, Rood PA and Kelley KW. (1988). J. Natl. Cancer Inst., 80, 1305-1312.

Martuza RL, Malick A, Markert JM, Ruffner KL and Coen DM. (1991). Science, 252, 854-856.

McNees AL and Gooding LR. (2002). Virus. Res., 88, 87-101.

Mineta T, Rabkin SD, Yazaki T, Hunter WD and Martuza RL. (1995). Nat. Med., 1, 938-943.

Moise AR, Grant JR, Vitalis TZ and Jefferies WA. (2002). J. Virol., 76, 1578-1587.

Moss B, Gershowitz A, Stringer JR, Holland LE and Wagner EK. (1977). J. Virol., 23, 234-239.

Mossman KL and Smiley JR. (2002). J. Virol., 76, 1995-1998.

Muster T, Rajtarova J, Sachet M, Unger H, Fleischhacker R, Romirer I, Grassauer A, Url A, Garcia-Sastre A, Wolff K, Pehamberger $\mathrm{H}$ and Bergmann M. (2004). Int. J. Cancer., 110, 15-21.

Najarro P, Traktman P and Lewis JA. (2001). J. Virol., 75, 3185-3196.

Nava VE, Cheng EH, Veliuona M, Zou S, Clem RJ, Mayer ML and Hardwick JM. (1997). J. Virol., 71, 4118-4122.

Ozoren N and El-Deiry WS. (2003). Semin. Cancer Biol., 13, 135-147.

Peters GA, Khoo D, Mohr I and Sen GC. (2002). J. Virol., 76, 11054-11064.

Poppers J, Mulvey M, Khoo D and Mohr I. (2000). J. Virol., 74, 11215-11221.

Rampino N, Yamamoto H, Ionov Y, Li Y, Sawai H, Reed JC and Perucho M. (1997). Science, 275, 967-969.

Ries SJ, Brandts $\mathrm{CH}$, Chung AS, Biederer $\mathrm{CH}$, Hann BC, Lipner EM, McCormick F and Korn WM. (2000). Nat. Med., 6, 1128-1133.

Roth W and Reed JC. (2002). Nat. Med., 8, 216-218.
Russell SJ. (2002). Cancer Gene Ther., 9, 961-966.

Sauthoff H, Heitner S, Rom WN and Hay JG. (2000). Hum. Gene Ther., 11, 379-388.

Shen Y, Kitzes G, Nye JA, Fattaey A and Hermiston T. (2001). J. Virol., 75, 4297-4307.

Smith GL, Howard ST and Chan YS. (1989). J. Gen. Virol., 70, 2333-2343.

Soengas MS, Capodieci P, Polsky D, Mora J, Esteller M, Opitz-Araya X, McCombie R, Herman JG, Gerald WL, Lazebnik YA, Cordon-Cardo C and Lowe SW. (2001). Nature, 409, 207-211.

Stojdl DF, Lichty B, Knowles S, Marius R, Atkins H, Sonenberg N and Bell JC. (2000). Nat. Med., 6, 821-825.

Tarodi B, Subramanian T and Chinnadurai G. (1994). Virology, 201, 404-407.

Telling G, Perera S and Williams J. (1994). J. Virol., 68, 541-547.

Todo T, Feigenbaum F, Rabkin SD, Lakeman F, Newsome JT, Johnson PA, Mitchell E, Belliveau D, Ostrove JM and Martuza RL. (2000). Mol. Ther., 2, 588-595.

Tollefson AE, Toth K, Doronin K, Kuppuswamy M, Doronina OA, Lichtenstein DL, Hermiston TW, Smith CA and Wold WS. (2001). J. Virol., 75, 8875-8887.

Verardi PH, Jones LA, Aziz FH, Ahmad S and Yilma TD. (2001). J. Virol., 75, 11-18.

Vogelstein B, Lane D and Levine AJ. (2000). Nature, 408, 307-310.

Wang Y, Hallden G, Hill R, Anand A, Liu TC, Francis J, Brooks G, Lemoine N and Kirn D. (2003). Nat. Biotechnol., 21, 1328-1335.

White E and Cipriani R. (1990). Mol. Cell Biol., 10, 120-130.

White E, Grodzicker T and Stillman BW. (1984). J. Virol., 52, 410-419.

White E, Sabbatini P, Debbas M, Wold WS, Kusher DI and Gooding LR. (1992). Mol. Cell Biol., 12, 2570-2580.

Wickham TJ. (2003). Nat. Med., 9, 135-139.

Yang J, Liu X, Bhalla K, Kim CN, Ibrado AM, Cai J, Peng TI, Jones DP and Wang X. (1997). Science, 275, 1129-1132.

Zhirnov OP, Konakova TE, Wolff T and Klenk HD. (2002). J. Virol., 76, 1617-1625. 